\section{Case Reports in Ophthalmology}

\title{
Bilateral Sequential NAION following Cataract Extraction: Case Report and Review of the Literature
}

\author{
Dana Barequet Elad Moisseiev Adi Michaeli Gad Dotan \\ Department of Ophthalmology, Tel Aviv Medical Center, and Sackler School of Medicine, \\ Tel Aviv University, Tel Aviv, Israel
}

\section{Key Words}

Nonarteritic ischemic optic neuropathy $\cdot$ Cataract surgery $\cdot$ Visual loss

\begin{abstract}
We report a 64-year-old patient who developed nonarteritic ischemic optic neuropathy (NAION) in both eyes following cataract extraction. The cataract surgeries in both eyes were uneventful and performed within a year, and NAION occurred a few months postoperatively in both eyes. A review of the literature on this rare complication is provided. This case report serves to raise awareness among cataract surgeons about this potential complication associated with irreversible visual loss, and especially about its high risk of bilaterality.
\end{abstract}

\section{Introduction}

C 2014 S. Karger AG, Basel

Cataract extraction is the most commonly performed ophthalmic surgery [1]. Although this procedure is not free of complications, neuroophthalmological adverse effects are rare and when they occur, they are more often related to the effects of retrobulbar anesthesia than to the surgery itself. Reported consequences include binocular diplopia secondary to the effects of the local anesthetic drug and traumatic optic neuropathy from direct trauma to the optic nerve during retrobulbar injection [2-4]. However, several publications described an association between cataract surgery and nonarteritic anterior ischemic optic neuropathy (NAION) [4-7], with an estimated incidence of 1 in 2,000 surgeries in patients aged >50 years, which is higher than the calculated risk for NAION in that age group [8]. Despite these publications, some uncertainty regarding this association still remains. Since cataract surgery is usually performed in elderly patients who may have vascular risk factors, it is possible that the occurrence of NAION after cataract extraction is purely coincidental based on the high performance rate of this surgery. Hayreh [9] divided NAION postcataract surgery into two types based on the time from surgery to the event. In the immediate type, ischemic

Elad Moisseiev, MD

Department of Ophthalmology, Tel Aviv Medical Center

Weitzman $6 \mathrm{St}$

Tel Aviv 64239 (Israel)

E-Mail elad_moi@netvision.net.il 
Barequet et al:: Bilateral Sequential NAION following Cataract Extraction: Case Report and Review of the Literature

damage to the optic nerve occurs within hours to days after surgery, occasionally after an episode of increased intraocular pressure occurring at the time of surgery or soon thereafter $[9,10]$, whereas in the delayed type, NAION ischemic optic nerve damage occurs months after surgery, often when recovery from surgery is already complete $[9,11]$.

Patients who had suffered from unilateral NAION and need to undergo cataract surgery in the other eye are often concerned that the same catastrophic event would occur once more in the unaffected eye, even if the first event did not occur after cataract surgery [12, 13]. The risk of second eye involvement in these circumstances has been reported to be higher than $50 \%$ [12].

In this case report we describe a patient who developed sequential NAION in both eyes following bilateral cataract extractions with normal operative and early postoperative course.

\section{Case Report}

A 64-year-old male was examined in the cataract clinic of the Tel Aviv Medical Center for the possible removal of a densely mature cataract in his right eye. His past medical history included heavy smoking, hypertension, hyperlipidemia, and noninsulin-dependent diabetes mellitus, and he did not have any past ocular history. Visual acuity (VA) in the right eye was hand movement and in the left eye 20/50. There was no relative afferent pupillary defect (RAPD). Visual loss in the right eye was consistent with the degree of maturity of the cataract. Since it was impossible to view the posterior pole through the dense cataract, ultrasonography was performed and found to be normal. The left eye had a nuclear sclerotic cataract and a normal dilated fundus examination. Subsequently, the patient underwent a cataract extraction in the right eye by phacoemulsification with an implantation of a posterior chamber intraocular lens. Surgery was performed under topical anesthesia and was uneventful. On the first day after surgery, corneal edema was seen, attributed to the high energy required to aspirate the dense cataract; however, it resolved rapidly during the following days. Intraocular pressure on the first postoperative visit was $15 \mathrm{~mm} \mathrm{Hg}$. One month after surgery, VA was 20/50 and the ocular examination was normal.

Two months after surgery, the patient experienced a sudden loss of vision in the right eye. Upon examination, VA was 20/120 and a positive right RAPD was found. The optic disc was swollen with surrounding flame-shaped hemorrhages. He was diagnosed with NAION and was started on aspirin $100 \mathrm{mg}$ daily.

Four months later, the patient complained of decreased vision in his left eye. VA in the left eye was 20/400 and was consistent with progression of the cataract. Following a detailed explanation of the risks involved with cataract surgery in these circumstances, including a $50 \%$ risk for NAION after surgery [12], the patient expressed his desire to undergo the procedure in order to improve his vision. Surgery in the left eye was uneventful, and 1 week after surgery VA in the left eye was 20/25. The early postoperative course during the first several months was entirely normal; however, 5 months after surgery the patient developed an abrupt loss of vision in his left eye. VA was reduced to finger counting, the pupils' reaction to light was sluggish, and the left optic disc was swollen with surrounding flame-shaped hemorrhages. Therefore, the visual loss was again attributed to the development of NAION.

On the patient's last follow-up examination 6 months after NAION had developed in the left eye and 10 months after its occurrence in the right eye, VA was 20/100 in the right eye and 20/200 in the left eye, with reduced color vision in both eyes. Anterior segment 
Barequet et al:: Bilateral Sequential NAION following Cataract Extraction: Case Report and Review of the Literature

examination was normal bilaterally. Right-trace RAPD was observed, and the optic nerves were atrophied and pale in both eyes (fig. 1). A 30-2 Humphrey visual field test revealed a severe depression with remnants of only the inferotemporal quadrant in the right eye and the superotemporal quadrant in the left eye (fig. 2a, b). Optical coherence tomography of the optic discs demonstrated bilateral thinning of the retinal nerve fiber layer (fig. 2c).

\section{Discussion}

In this case report we present a patient who had bilateral sequential delayed-type NAION following uncomplicated cataract extraction. The mechanism of pathogenesis causing delayed-type NAION after cataract extraction is controversial and has not been clearly elucidated [9-14]. The most common hypothesis explaining this complication is vasculopathy related to intraocular inflammation. It has been suggested that the surgical insult causes an inflammatory reaction, which may cause optic disc edema and ischemic damage resulting in NAION weeks to months after surgery [15-17]. Although we cannot be certain that the cataract surgery had led to the development of NAION in our patient, the sequence of events raises suspicion regarding this possibility. Previous case reports have described NAION following cataract extraction; however, our report is unique since it describes the occurrence of NAION following sequential and uneventful phacoemulsification in both eyes. Previous publications provide further support to the association between cataract surgery and NAION [4-7]. McCulley et al. [7] reviewed 18 cases of NAION that developed within 1 year after cataract surgery and found that all cases occurred during the first 6 months after surgery, most of them during the first weeks. The authors argued that this time distribution supports the relationship between cataract surgery, and optic nerve insult since spontaneous NAION unrelated to cataract surgery would be expected to occur randomly during the first year, not showing a time-based proximity to the surgery. In a different study by the same group [14], it was found that patients who had had NAION after cataract surgery had a much lower chance of having a small cupless disc in the uninvolved eye ('disc at risk'), which is a common feature found in patients with spontaneous NAION. In this study it was also found that hypertension, which is commonly found in patients with spontaneous NAION, is not a risk factor for NAION after cataract surgery [14]. These findings suggest that NAION truly is a possible complication of cataract surgery, even if its occurrence is not in a temporal proximity to surgery. Lam et al. [12] have found that, in patients who had previously suffered from unilateral NAION, the risk of developing the same condition in the initially uninvolved eye is 3.6 times higher after cataract surgery than in unoperated eyes. The risk of NAION in operated eyes was 53\%, which is much higher than the $19 \%$ risk of second eye involvement in unoperated eyes. This increased risk following surgery was found to be independent of any other risk factors [12].

Our patient, who suffered from NAION following cataract extraction in one eye, was warned about the risk for a similar event following surgery in the other eye; however, he decided that surgery was necessary and unfortunately, 5 months after the surgery, he developed the same condition in the other eye as well. In both eyes, NAION occurred despite uneventful surgery and a normal early postoperative course, without any intraocular pressure elevation. The series of events in this case illustrates the potential for significant visual loss due to the rare complication of NAION following cataract extraction, even when the surgery and postoperative period are uneventful, as well as the high risk for bilateral involvement. We acknowledge that our patient had diabetes, hypertension and was a heavy smoker, factors which are all associated with an increased risk of cardiovascular events and 
Barequet et al.: Bilateral Sequential NAION following Cataract Extraction: Case Report and Review of the Literature

which may have predisposed him to NAION. However, the close temporal proximity between the cataract extraction and NAION, that was repeated bilaterally, suggests a causative effect of the procedure.

Although it is impossible to completely prevent the occurrence of NAION following cataract surgery, the performing surgeons should be aware of this potential complication, which is often associated with permanent visual loss. It is especially important to recognize the increased risk in patients who had already suffered from NAION in the other eye. A preoperative discussion explaining these possible consequences is necessary. It is also recommended that in these circumstances, cataract surgery should be performed by a welltrained surgeon who is familiar with the risks involved and in a setting that allows regular monitoring of the intraocular pressure during the first hours after surgery. A prevention of intraocular pressure elevation in the first days after surgery may prevent the occurrence of early postoperative NAION, but probably does not affect late-onset disease, as it has occurred in our patient.

\section{Disclosure Statement}

The authors have no proprietary interests to disclose.

\section{References}

1 Rutkow I: Surgical operations in the United States: then (1983) and now (1994). Arch Surg 1997;132:983990.

2 González N, Quintana JM, Bilbao A, et al: Factors affecting cataract surgery complications and their effect on the postoperative outcome. Can J Ophthalmol 2014;49:72-79.

3 Kurt E, Mayalı H: Early post-operative complications in cataract surgery; in Zaidi F (ed): Cataract Surgery. InTech, Rijeka, 2013, pp 245-258.

4 Lee MS, Rizzo JF, Lessell S: Neuro-ophthalmologic complications of cataract surgery. Semin Ophthalmol 2002;17:149-152.

5 McCulley TJ, Lam BL, Feuer WJ: Incidence of nonarteritic anterior ischemic optic neuropathy associated with cataract extraction. Ophthalmology 2001;108:1275-1278.

6 Lee H, Kim CY, Seong GJ, Ma KT: A case of decreased visual field after uneventful cataract surgery: nonarteritic anterior ischemic optic neuropathy. Korean J Ophthalmol 2010;24:57-61.

7 McCulley T, Lam B, Feuer W: Nonarteritic anterior ischemic optic neuropathy and surgery of the anterior segment: temporal relationship analysis. Am J Ophthalmol 2003;136:1171-1172.

-8 Johnson L, Arnold A: Incidence of nonarteritic and arteritic anterior ischemic optic neuropathy: populationbased study in the state of Missouri and Los Angeles County, California. J Neuroophthalmology 1994;14:3844.

-9 Hayreh S: Anterior ischemic optic neuropathy. IV. Occurrence after cataract extraction. Arch Ophthalmol 1980;98:1410-1416.

10 Serrano L, Behrens M, Carroll F: Postcataract extraction ischemic optic neuropathy. Arch Ophthalmol 1982;100:1177-1178

11 Townes CD, Moran CT, Pfingst HA: Complications of cataract surgery. Am J Ophthalmol 1952;35:1311-1319.

-12 Lam BL, Jabaly-Habib H, Al-Sheikh N, et al: Risk of non-arteritic anterior ischaemic optic neuropathy (NAION) after cataract extraction in the fellow eye of patients with prior unilateral NAION. Br J Ophthalmol 2007;91:585-587.

13 Beri M, Klugman M, Kohler J, Hayreh SS: Anterior ischemic optic neuropathy. VII. Incidence of bilaterality and various influencing factors. Ophthalmology 1987;94:1020-1028.

14 McCulley TJ, Lam BL, Feuer WJ: A comparison of risk factors for postoperative and spontaneous nonarteritic anterior ischemic optic neuropathy. J Neuroophthalmol 2005;25:22-24.

15 Gass JD, Norton EW: Cystoid macular edema and papilledema following cataract extraction: a fluorescein fundoscopic and angiographic study. Arch Ophthalmol 1966;76:646-661.

16 Slavin ML, Lopinto RJ, Prywes AS, Rosen DA: Optic disk edema with aphakic cystoid maculopathy masquerading as ischemic optic neuropathy. J Clin Neuroophthalmol 1985;5:180-184.

17 McCulley TJ: Ischemic optic neuropathy and cataract extraction: what do I need to know? Oman J Ophthalmol 2012;5:141-143. 


\section{Case Reports in \\ Ophthalmology}

\begin{tabular}{l|l}
\hline Case Rep Ophthalmol 2014;5:292-296 \\
\hline DOI: 10.1159/000365913 & $\begin{array}{l}\text { ○ 2014 S. Karger AG, Basel } \\
\text { www.karger.com/cop }\end{array}$ \\
\hline
\end{tabular}

Barequet et al.: Bilateral Sequential NAION following Cataract Extraction: Case Report and Review of the Literature
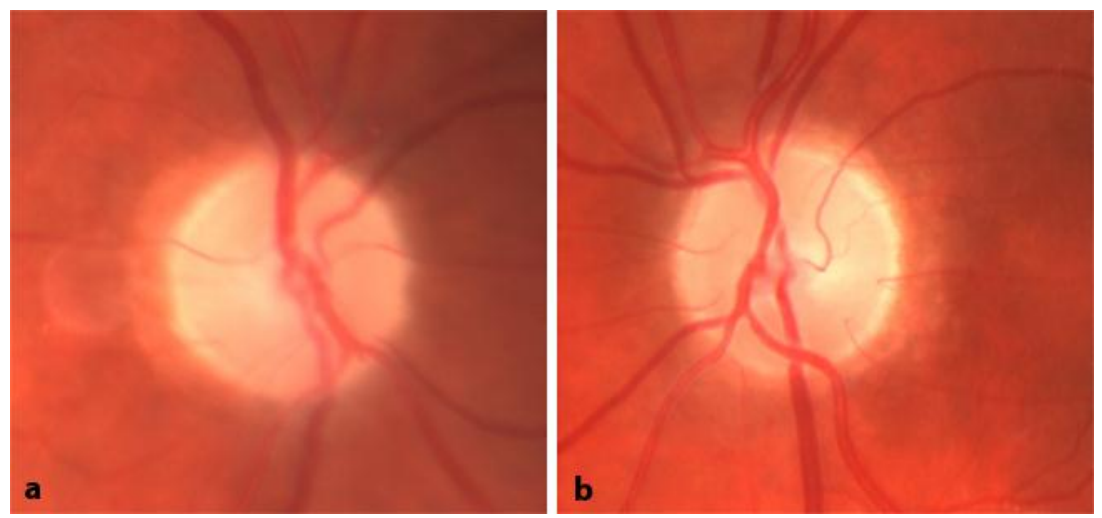

Fig. 1. Enlarged view of the optic discs demonstrating their pallor, consistent with the diagnosis of bilateral NAION. a Right eye. b Left eye.

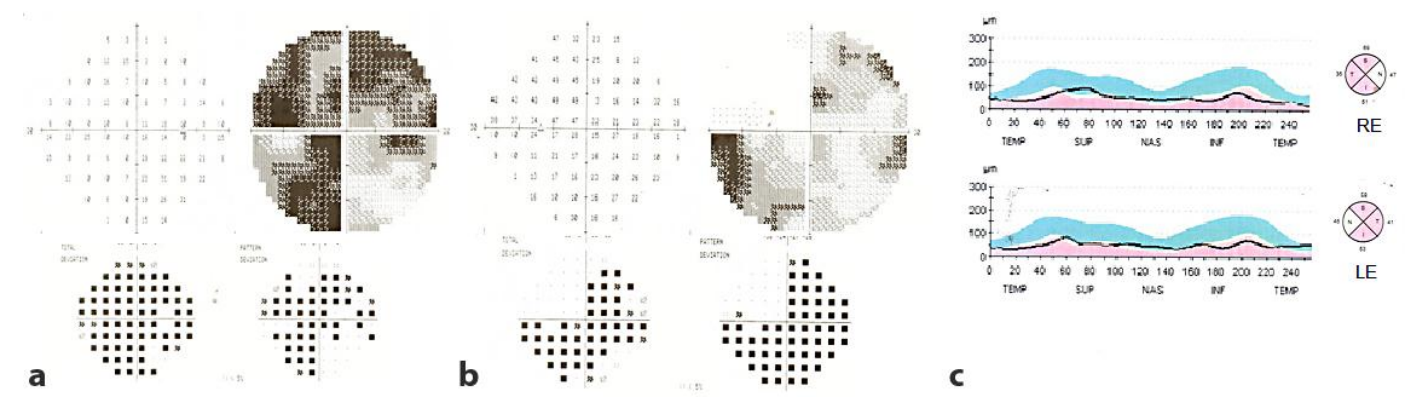

Fig. 2. a, b 30-2 Humphrey visual field test with grayscale, total deviation and pattern deviation scales are presented. a The right eye had a mean deviation of $-20.4 \mathrm{~dB}$, showing a severe depression with remnants of only the inferotemporal quadrant. $\mathbf{b}$ The left eye had a mean deviation of $-8.2 \mathrm{~dB}$, showing a severe depression with remnants of only the superotemporal quadrant. c Optical coherence tomography of the retinal nerve fiber layer demonstrated a moderate loss of nerve fibers in the nasal quadrant and a severe loss of nerve fibers in the other quadrants, corresponding to the visual field test. 